\title{
Switchable Chiroptical Hot-Spots in Silicon Nanodisk Dimers
}

Xin Zhao and Björn M. Reinhard

Department of Chemistry and The Photonics Center, Boston University, Boston, MA 02215, United States

Supporting Information 
a)

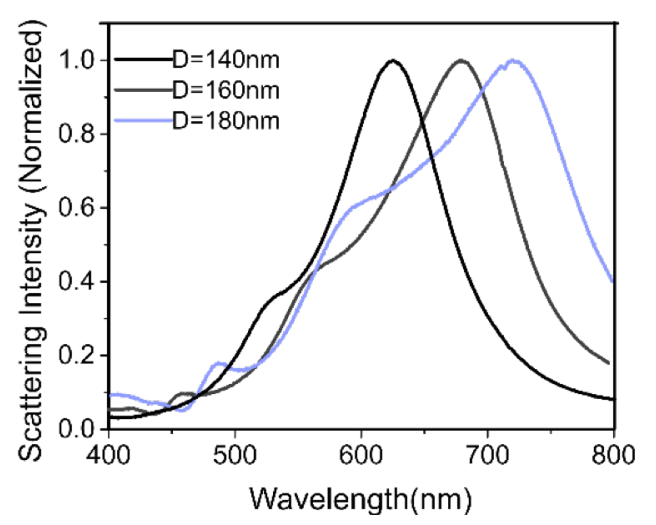

b)

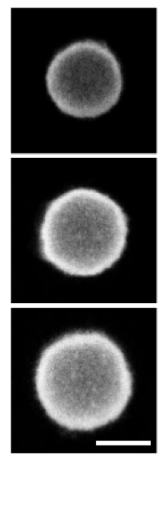

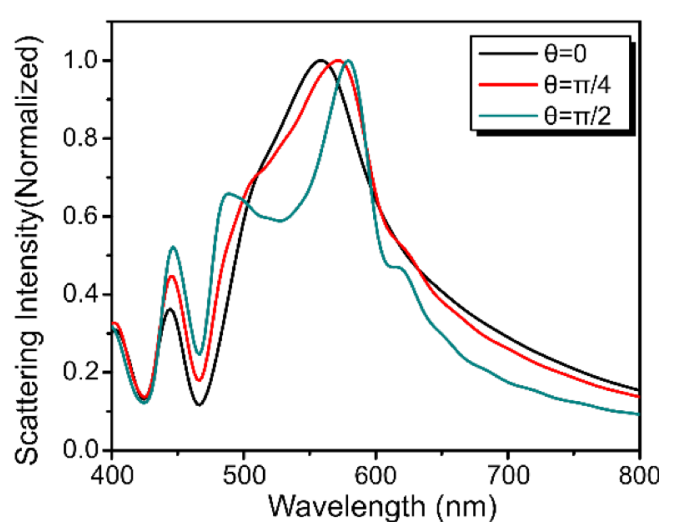

Figure S1. a) Experimental darkfield scattering spectra for Si nanodisk monomers ( $h=150$ $\mathrm{nm}$ ) with different diameters $D$ fabricated on sapphire substrate. b) Simulated scattering spectra for free-standing (no substrate) silicon nanodisk dimer ( $D=140 \mathrm{~nm}, h=100 \mathrm{~nm}, g=$ $20 \mathrm{~nm}$ ) for different light polarization angles.

a)

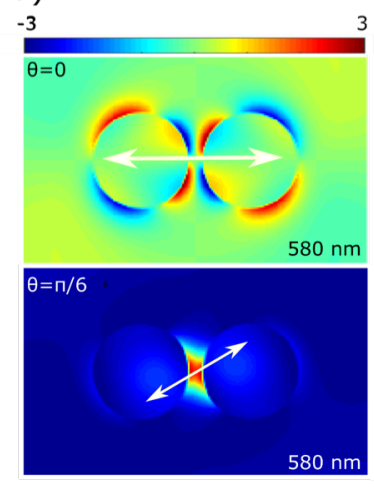

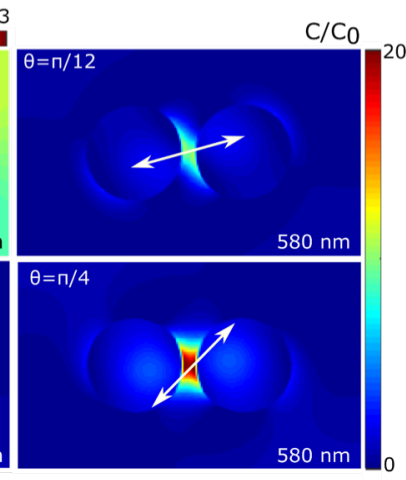

b)

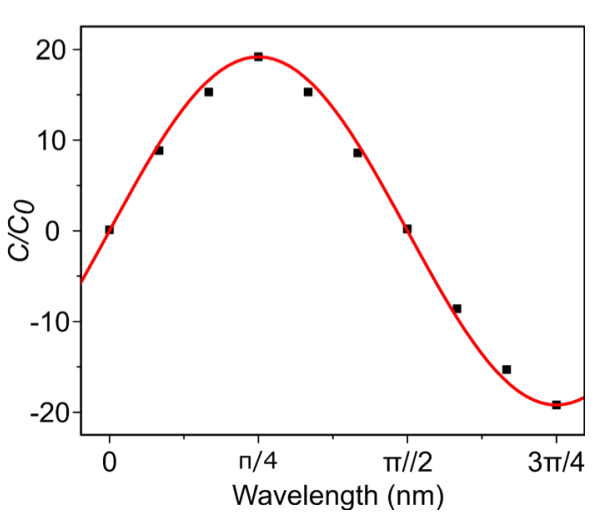

Figure S2. a) Effect of polarization angle on optical chirality enhancement. Simulated optical chirality enhancement $C / C_{0}$ for Si nanodisk dimer $(h=100 \mathrm{~nm}, g=20 \mathrm{~nm}, D=140$ $\mathrm{nm}$ ) with different polarization angles (clockwise top left to bottom left) $\theta=0, \pi / 12, \pi / 4$, $\pi / 6$. b) Plot of the calculated $C / C_{0}$ (integrated over the gap area) as function of polarization angle $\theta$. 


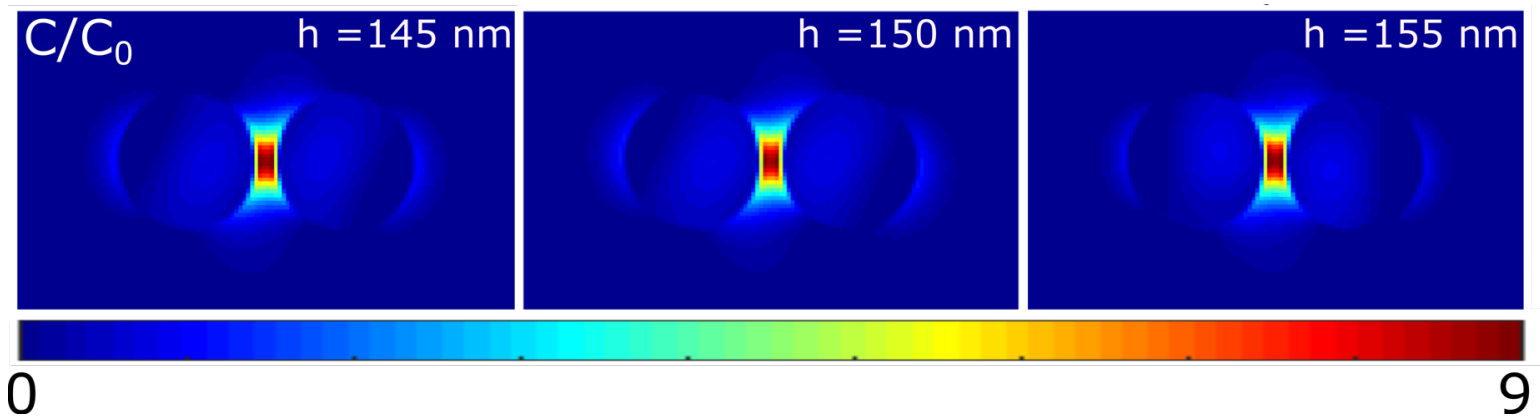

Figure S3. Height effect on optical chirality. Simulated optical chirality enhancement for $D=$ $140 \mathrm{~nm}, g=20 \mathrm{~nm}$ Si nanodisk dimers on sapphire substrate with different heights.

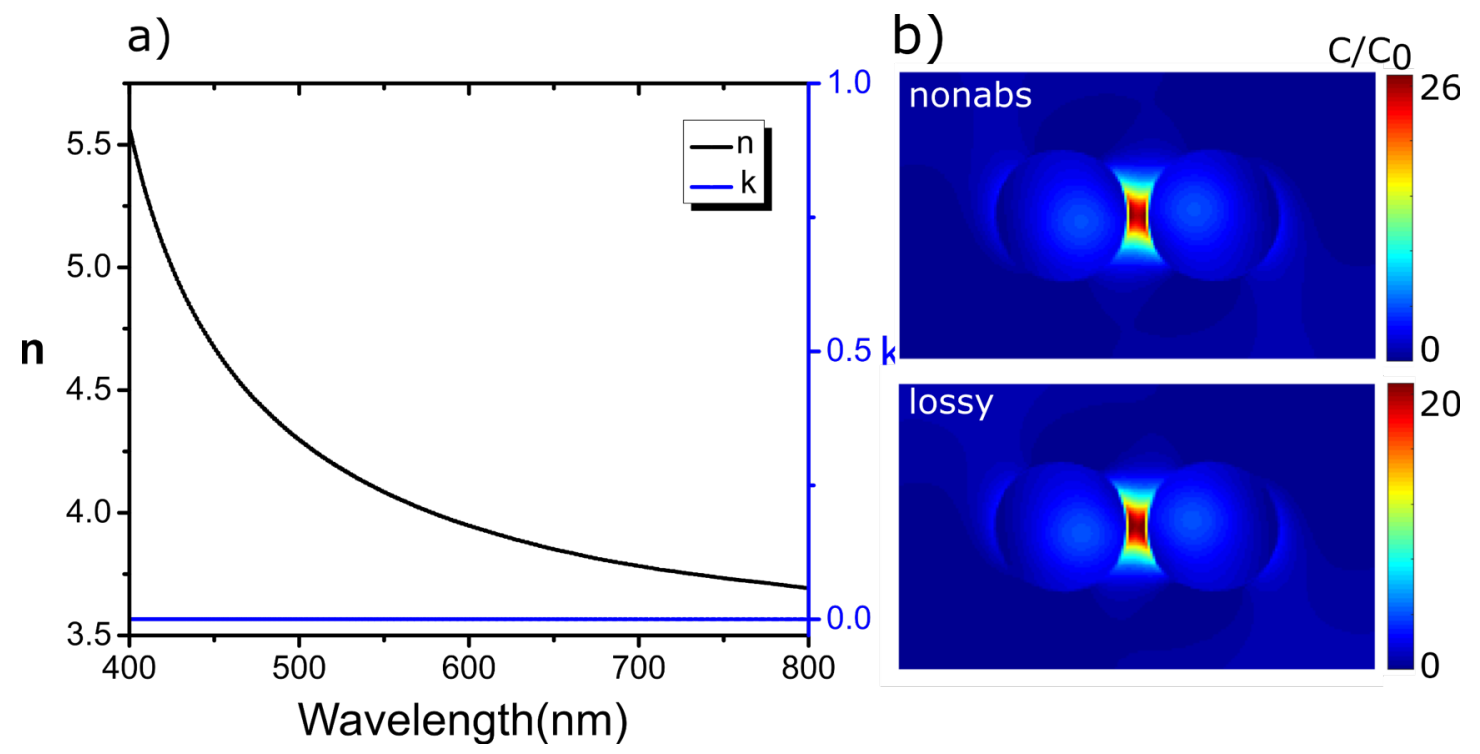

Figure S4. Simulated optical chirality enhancement by hypothetical non-absorbing Si. a) Wavelength-dependent refractive index. b) Optical chirality maps for non-absorbing dimers ( $h=100 \mathrm{~nm}, g=20 \mathrm{~nm}, D=140 \mathrm{~nm}$ ) when in vacuum (upper) and a comparison of this $C / C_{0}$ map with that for the "lossy" Si nanodisk dimer (bottom) shows a nearly identical spatial distribution of $C$ enhancement with higher peak enhancement $\left(C / C_{0}=26\right.$ compared to $C / C_{0}=$ 20). 